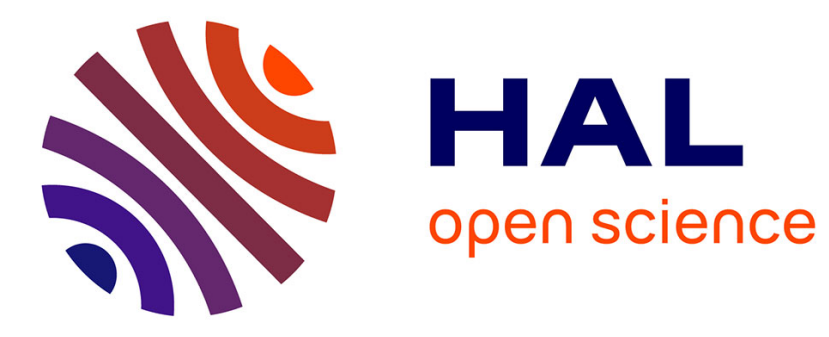

\title{
Use of extensive habitat inventories in biodiversity studies
}

\author{
Audrey Muratet, Emmanuelle Porcher, Jean-Claude Abadie, Laurent Poncet, \\ Jacques Moret, Alexandre Robert
}

\section{- To cite this version:}

Audrey Muratet, Emmanuelle Porcher, Jean-Claude Abadie, Laurent Poncet, Jacques Moret, et al.. Use of extensive habitat inventories in biodiversity studies. Biodiversity and Conservation, 2009, 18 (12), pp.3115-3125. 10.1007/s10531-009-9630-6 mnhn-02265719

\section{HAL Id: mnhn-02265719}

\section{https://hal-mnhn.archives-ouvertes.fr/mnhn-02265719}

Submitted on 12 Aug 2019

HAL is a multi-disciplinary open access archive for the deposit and dissemination of scientific research documents, whether they are published or not. The documents may come from teaching and research institutions in France or abroad, or from public or private research centers.
L'archive ouverte pluridisciplinaire HAL, est destinée au dépôt et à la diffusion de documents scientifiques de niveau recherche, publiés ou non, émanant des établissements d'enseignement et de recherche français ou étrangers, des laboratoires publics ou privés. 
Biodiversity studies

1

2

3

4 Muratet, Audrey ${ }^{1 *}$; Porcher, E. ${ }^{1,2}$ Abadie, J-C. ${ }^{1,2}$; Poncet, L. ${ }^{1}$; Moret, J. ${ }^{1}$ \& Robert, 5

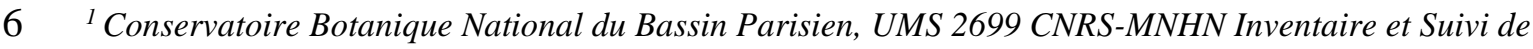

7 la Biodiversité, Muséum national d'Histoire naturelle, 61 rue Buffon F-75005 Paris, France; ${ }^{2}$ UMR

85173 CNRS-MNHN-UPMC, Conservation des Espèces, Restauration et Suivi des Populations, Muséum

9 national d'Histoire naturelle, 61 rue Buffon F-75005 Paris, France; * Corresponding author ; Fax +33 1

10407935 53; Email muratet@mnhn.fr 


\section{Abstract}

Large monitoring programs exist in many countries and are necessary to assess

16 present and past biodiversity status and to evaluate the consequences of habitat

17 degradation or destruction. Using such an extensive data set of the floristic richness in

18 the Paris Ile-de-France region (France), we compared different sampling efforts and

19 protocols in different habitat units to highlight the best methods for assessing the actual

20 plant biodiversity.

Our results indicate that existing data can be used for a general understanding of

22 site differences, but analysts should be aware of the limitations of the data due to non-

23 random selection of sites, inconsistent observer knowledge, and inconsistent sampling period. The average species diversity recorded in a specific habitat does not necessarily reflect its actual diversity, unless the monitoring effort was very strong.

Overall, increasing the sampling effort in a given region allows improvement of

27 the (i) number of habitats visited, (ii) the total sampled area for a given habitat type, (iii)

28 the number of seasons investigated. Our results indicate that the sampling effort should

29 be planned with respect to these functional, spatial and temporal heterogeneities, and to 30 the question examined. While the effort should be applied to as many habitats as

31 possible for the purpose of capturing a large proportion of regional diversity, or 32 comparing different regions, inventories should be conducted in different seasons for 33 the purpose of comparing species richness in different habitats. 
Biodiversity studies

37 Keywords: data quality; floristic diversity; monitoring; sampling effort; species

38 richness

39

40 


\section{Introduction}

It is now widely recognized that the current extinction rates of plant and animal species are between a hundred and a thousand times higher than the background rates throughout life's history on Earth (May 2002). However, documenting species extinction only, i.e. the most obvious manifestation of biodiversity loss, is not sufficient to develop effective conservation policies, partly because extinction rates carry no information regarding changes in community composition, which may have dramatic consequences for ecosystem stability (Worm et al. 2003). There is an urgent need to quantify the spatiotemporal changes in biodiversity by considering community composition and trends in species abundances (Convention on Biological Diversity in Rio, 1992). Such information is necessary to identify the mechanisms (e.g. environmental variables, human-induced disturbances, etc.) controlling the variation in species richness through space and time, as well as to identify sites of conservation concern and appropriate policies to improve the current biodiversity.

Ideally, this quantification would require large scale, long-term surveys based on standardized methodologies to allow comparisons in space and time. Such protocols already exist in a limited number of cases or are just starting to be implemented. The British Countryside Survey (CS) (Firbank et al., 2003; Haines-Young et al., 2003), for example, was established in 1978 in the United Kingdom and focuses on several

60 taxonomic groups, including plants. The Biodiversity Monitoring Program (BDM) in

61 Switzerland (Weber et al., 2004; Plattner et al., 2004) was launched in 1995 and focuses on local plant diversity. Other protocols have been implemented to survey the diversity

63 of particular taxonomic groups, as exemplified by breeding bird surveys in different 64 countries (since 1966 in North America, Sauer et al. 1997; since 1994 in UK, Newson et 
Biodiversity studies

65

66

67

68

69

al. 2005; since 1989 in France, Julliard et al. 2003). Such surveys are based on formatted sampling protocols generally occurring twice a year within different discrete classes of habitat at the national scale. In these examples, the inventory protocol is generally standard and well defined, which allows the sampling effort to be homogeneous among observers, constant in time, or clearly quantified, so that any statistical inference can be made independently of the monitoring effort. Moreover, inventory protocols are designed to ensure that sampling is proportional to the area occupied by each habitat / settlement type in the region of interest.

Although such large scale monitoring schemes are crucial to document future changes in biodiversity, they will unfortunately not suffice to quantify the present changes in biodiversity, and specifically to evaluate the 2010 biodiversity target. A complementary approach to quantify changes in biodiversity could be to use the large amounts of existing inventory data collected by various biodiversity stakeholders (some of which are compiled in the Global Biodiversity Information Facility, GBIF 2008). However, because such data come from a very large number of observers and geographic locations, they were generally collected using very different methodologies and are highly heterogeneous in nature. The question that immediately arises is whether such heterogeneous data can be exploited to document reliably the trends in biodiversity.

Here we address this issue using plant inventory data from Paris Basin (France).

We analyzed data from thousands of inventories carried out between 2001 and 2005 by botanists who were involve in the same Botanical Conservatory but who were not instructed to follow a given standardized protocol. Focusing on the proportion of total 
Biodiversity studies

89 vascular plant species detected as a function of (1) annual number of visits per habitat

90 type and (2) season of data collection, we investigated different options for data analysis

91 and survey protocol, to optimize the use of existing data and improve future monitoring.

92 We specifically addressed the following questions: 1) Are one time surveys of floristic

93 diversity indicative of the total diversity of a region, and do species richness estimated

94 from one time surveys vary across habitats, seasons and years? 2) What is the benefit of

95 increasing survey effort, by increasing either the number of survey habitats or the time

96 span of surveys?

97 


\section{Material and methods}

\section{Study area}

100 The Ile-de-France region, including the city of Paris $\left(48^{\circ} 68^{\prime} \mathrm{N} ; 0^{\circ} 17^{\prime} \mathrm{E}\right)$ and the

101 surrounding area, covers $12,072 \mathrm{~km}^{2}$ (Fig. 1). The climate is oceanic with continental

102 trends (mean annual temperature $12{ }^{\circ} \mathrm{C}$, with a minimum in January and a maximum in 103 July; average monthly rainfall $57 \mathrm{~mm}$ ) and the relief is relatively flat (elevation between

10411 and $217 \mathrm{~m}$ a.s.1.). The population density is 952 inhabitants $/ \mathrm{km}^{2}$ (INSEE 2006),

105 which makes Ile-de-France the most densely populated administrative region of France.

106 A total of 1225 plant species were encountered in the study area between 2001

107 and 2005, as calculated from records of the FLORA database (National Botanical

108 Conservatory of the Paris Basin, CBNBP 2008 and see below for a description of the

109 database). Of these species, $11 \%$ were naturalized species, i.e. non-indigenous species

110 that reproduce and sustain populations without direct intervention by humans

111 (Richardson et al. 2000).

113 Inventory protocol

114 The data used in this study were collected between 2001 and 2005 by botanists

115 from the National Botanical Conservatory of the Paris Basin (hereafter CBNBP), a

116 French public organization aiming to study and protect the flora of the Paris basin. One

117 central objective of CBNBP is to describe the geographical distribution of all species

118 growing in the area, which dictates the methodology used to collect data. Every year, a

119 total of 149 botanists (both professionals and competent amateurs) visited the

120 'communes' (French administrative municipalities) of the region between March and

121 October and recorded as many plant species as they could observe within a 
Biodiversity studies

122 municipality, as well as the spatial locations of each species. There was no standardized

123 protocol: the duration of data collection, sampling locations and total area sampled were

124 left to the appreciation of the observers and varied greatly among individuals. For

125 example, sampling locations within a municipality were not randomly distributed, but

126 were instead usually chosen to maximize the total number of species observed.

127

128 Database contents and study data

129 Inventory data were pooled in FLORA, a database built by CBNBP. The

130 database includes information on species (scientific and common names), observer, date

131 of observation, location (municipality) and habitat type according to CORINE land

132 cover nomenclature (Bissardon et al. 1997), and contains more than one million

133 observations (i.e. one species recorded at a given time and in a given site) for the Ile-de-

134 France region (CBNBP 2008).

135 We chose to work with data collected between 2001 and 2005, because the

136 quality and quantity of data are much lower before this period. For statistical reasons,

137 we also discarded all observations from rarely sampled habitats, i.e. habitats that were

138 visited less than once a month between 2001 and 2005, so that data from eight habitat

139 types only were retained (see Table 1). For this study, this yielded a total of 237,884

140 observations corresponding to 7,358 different sites (i.e. the total area covered by a given

141 habitat type in a given place) within the Ile-de-France region.

142

143 Data analysis 
Biodiversity studies

144 Because the database contains very little information on species abundance or 145 frequency, and does not allow estimating species detection probabilities, plant

146 communities were characterized by the observed species richness only.

148 Species richness at the site level

149 We first analyzed the variation in species richness at the site level by fitting an 150 analysis of variance model using the R software (Core Team 2007), where site richness was a function of (1) habitat type (2) inventory month, (3) inventory year, and (4) all pairwise interactions.

As this analysis showed statistical differences among years on the richness

154 recorded, all years were considered separately in subsequent analysis.

Assessment of optimal monitoring effort

To optimize monitoring programs, monitoring effort should be minimum, but large enough to provide accurate estimates of species richness (and, ideally, other parameters of community composition). To evaluate this, we performed random resampling in the database to simulate various monitoring efforts, by varying the

161 number of sites, habitats, or months sampled.

a) Increasing effort within a given habitat

To estimate the species accumulation curve within each habitat type, we

164 plotted the ratio of observed vs. total species richness as a function of the number of inventories, $x$, as follows. Within a given year, $x$ inventories ( $=x$ sites $)$ were sampled at

166 random, each in a different month, and the overall species richness (excluding

167 redundancies) of this sample was computed. This species richness was then divided by

168 the total number of species observed in this habitat type. For each $x$ and each habitat 
Biodiversity studies

169 type, the procedure was repeated 50,000 times and the average ratio of observed vs.

170 total species richness was plotted.

171

172

b) Correlation between sampled and total species richness

173

To test whether the number of species recorded in $x$ inventories was

174 representative of the "true" floristic richness of the different habitats, we compared the number of species recorded in $x$ inventories within a year in each habitat to the overall number of species in each habitat, using a Spearman rank correlation across habitats.

177 This procedure was performed 50,000 times for each habitat, and the average 178 correlation coefficient, $r_{s}$, as well as the proportion of significant correlations at the 5\%

179 level were plotted as a function of the number of inventories per habitat, $x$.

c) Optimization of the number of habitats or months sampled

182 habitats sample, given a constant effort. To this end, we plotted the observed species

183 richness as a function of number of habitats (respectively months) visited, with a

184 constant number of inventories. Keeping the number of inventories (8) constant allowed us to test for a habitat or month effect without confounding area effects. Within a given year, eight sites were chosen at random among $x$ habitat types (respectively months) and

187 the overall species richness in these eight inventories (i.e., excluding redundancies) was

188 computed. The procedure was repeated 50,000 times and the average species richness in

$189 x$ habitats (respectively months) was plotted against the number of habitats (months). 
191 Results

\section{Variation in average observed species diversity}

193 Site species richness varied significantly across years, months (maximum

194 species richness in June (36.5), minimum in August (28)), and habitat types (maximum

195 number of species in cities and industrial sites (41), minimum in stagnant freshwater

196 (18), Table 2). In addition, all interactions were also significant, so that the difference in

197 species richness among habitats were highly variable within and across years (Table 2).

198

199 Species accumulation curves within habitats

200 The shape of the species accumulation curves varied greatly across habitats (Fig.

201 2). The proportion of total species recorded appeared to reach a plateau at five inventories per habitat in mesophile meadows, cultures, cities and industrial sites or wastelands. Note however that the fraction of total species observed remained low (between 15 and 25\%). In contrast, the species accumulation curves did not appear to saturate in stagnant fresh water, circle of water edges, deciduous forest or urban parks and gardens.

\section{Correlation between observed and total species richness across habitats}

As expected, the correlation between observed and total species richness across

210 habitats was close to zero and non-significant when the number of inventories per

211 habitat was small ( $x<6$, Fig 3). However, seven or eight inventories per habitat

212 provided a better picture of the total species richness (Spearman correlation coefficient

213 significantly different from 0, Fig. 3a). Note however that mean correlation coefficients

214 remained relatively low (fig 3a), suggesting that yearly monitoring protocols with few 
Biodiversity studies

215 inventories in each habitat do not allow to compare species richness in the different 216 habitats.

217

218 Optimization of monitoring effort by increasing the number of habitats or months As expected, observed species richness increased $(+12 \%)$ when the number of

220 habitats increased for a constant monitoring effort. Similarly, there was a lower but non 221 negligible benefit $(+7 \%)$ of increasing the number of inventory months.

222

223 


\section{Discussion}

Biodiversity inventories are costly in time and money, and maximizing the number of species observed during a given monitoring effort is therefore an important task. Our study focuses on the use of existing, non standardized inventory data to address the optimization of monitoring effort.

\section{Non-standardized data and minimal monitoring effort}

Our results reflect the well-known heterogeneity of plant communities in time (year) and among habitat types: the observed species richness depends on the habitat, season, year and their interactions. When dealing with non-standardized data, this raises the issue of how to disentangle actual ecological sources of heterogeneity (e.g. true differences among habitat, seasons, years...) from sampling or methodological sources of variation. In particular, owing to the lack of randomization and to observer variability, among-inventory differences in species richness were not only due to differences in the period of sampling (month and year), but also to differences in sites themselves (inventories performed in different months were not necessarily conducted on the same sites). This for example implies that classical methods to estimate species richness (e.g. those derived from the CAPTURE program, Rexstad and Burnham 1991) cannot be used with such non-standardized inventory data. Hence, total species richness in a given habitat was estimated as the total number of species observed over a large number of inventories. Although this probably results in an underestimation of species richness, we nonetheless believe that it provided a reasonably good picture of community composition. 
Biodiversity studies

247 General guidelines about minimal monitoring efforts can be inferred from the results

248 above. We showed that one to five yearly inventories per habitat do not provide an

249 accurate picture of habitat richness (Fig. 4), at least in the semi-natural habitats

250 commonly encountered in Île-de-France. Sampling effort is clearly an important issue

251 regardless of the survey method used (Metcalfe-Smith et al. 2000; Walther \& Martin

252 2001), and other studies have reached similar conclusions regarding minimal sampling

253 efforts. For example, De Solla et al. (2005) showed that, in anuran monitoring

254 programs, the average observed species richness was only $25.1 \%$ of the total richness

255 with a single sampling night, but reached an average of $80 \%$ of the total species

256 richness with 12 sampling nights. Archaux et al. (2006) showed that on $400 \mathrm{~m}^{2}$ forest

257 quadrats, the level of exhaustiveness of plant censuses increased in a semi-logarithmic

258 way with sampling time. The study of Estevez \& Christman (2006) on the movement of

259 animals in confinement clearly indicated that sampling effort had a tremendous impact

260 on the study outcome. Nonetheless, several European countries have started to

261 implement floristic monitoring programs, generally based on one or two inventories per

262 year. For instance, in the United Kingdom, the British Countryside survey (Haines-

263 Young et al. 2000) is based on annual inventories of several hundred of randomly

264 sampled fix plots classified into 32 land use classes. In Switzerland, The Biodiversity

265 Monitoring Program (Hintermann et al. 2002) consists in a grid-sampling program

266 based on five settlement types within which plots are randomly drawn. The local plant

267 diversity is inventoried in these plots every five years.

268 Although the information collected in the aforementioned monitoring programs is

269 useful to document long-term trends, or to compare trends among habitat types

270 (especially for the most frequent species, and when directional variations in species 
Biodiversity studies

271 abundances are high), our results suggest that it will not be sufficient to compare the

272 absolute species numbers present in the different habitat types. In the present data set,

273 the variability across observers and sites tended to overwhelm the differences among

274 habitat types when there were fewer than six inventories per year (Fig. 4), which

275 represents a large monitoring effort in comparison with most survey programs.

276

277 Optimization of sampling effort

278 The outcome of a given protocol depends, among others, on the area sampled as

279 well as on seasonal and habitat effects, so that the sampling effort should be judiciously

280 planned and implemented to optimize the number of species recorded. In general, the

281 financial and time costs of a field inventory do not vary across seasons or habitat types

282 and protocols can be optimized via a selection of seasons and habitats visited. For

283 example, with a constant effort, the observed species richness was increased by $6.5 \%$ if

284 inventories were conducted in two different seasons vs. a single season, and by $11 \%$ if

285 they were conducted in two vs. one habitats. This is consistent with the generally

286 accepted idea that plant functional beta diversity is larger than seasonal beta diversity.

287 However, the choice of maximizing either the number of seasons or habitats sampled

288 should depend on the question investigated.

289 If a monitoring program aims to maximize recorded species richness in the study

290 region (e.g. for the purpose of comparing biodiversity across regions or examining

291 annual trends), maximizing habitat types would be the most efficient strategy. In fact,

292 our results indicate that (i) increasing the number of habitats is always more efficient

293 than increasing the number of months; (ii) beyond three months, any further increase in

294 the number of months sampled has no notable effect on the observed species richness 
Biodiversity studies

295 for a constant number of sites visited (Fig.4). In contrast, to compare species richness 296 across habitats, inventories should be conducted throughout as many sites as possible to

297 ensure that actual differences among habitats can be detected. Assuming that the total 298 species richness was a proxy for true total species richness, we showed that the average 299 species richness observed during a single inventory per habitat was not representative of 300 total richness. First, the average species richness observed in a single inventory was only $4.24 \pm 2.84 \%$ of total richness on average. Second, (b) observed richness is not representative of the total richness of the habitat unless the sampling effort is extremely strong (> 5 inventories a year, figure 3). It follows that for a constant sampling effort,

304 among habitat comparisons require to use few habitats with many inventories per 305 habitat.

\section{Conclusion}

There is general agreement that biodiversity conservation should be guided by

310 biodiversity assessment. As an important part of this assessment, inventory protocols

311 should be designed with care, to identify the specific conservation target that a project

312 ultimately would like to influence (Salafsky et al. 2002). Ideally inventories should

313 include (1) sites randomly sampled according to a standard protocol (for example, using

314 a sampling effort stratified by habitat types), (2) observers with a knowledge level as

315 uniform as possible (3) identical observation periods. As we promote these goals we

316 will promote high quality data for monitoring and other purposes. Existing large data

317 sets collected by various biodiversity stakeholders do not generally meet these criteria,

318 and they should be used with caution to infer biodiversity trends, e.g. in combination 
Biodiversity studies

319 with resampling methods to correct for their heterogeneity. The large number of 320 existing inventory data can however be exploited to address other conservation issues,

321 e.g. to quantify floristic index over a homogeneous region (Muratet et al. 2008).

322

323 
Biodiversity studies

\section{Acknowledgments}

325 We thank Gérard Arnal and Sébastien Filoche of the CBNBP, coordinators of the

326 floristic inventories in the Paris Ile-de-France region. Emmanuelle Porcher was partly

327 funded by Agence Nationale de la Recherche (ANR Grant \#2006-JCJC- 0032).

328 
Biodiversity studies

\section{References}

330 Archaux F, Gosselin F, Bergès L (2006) Effects of sampling time, species richness

331 and observer on the exhaustiveness of plant censuses. J. Veg. Sci. 17: 299-306.

332 Bissardon M, Guibal L, Rameau J-C (1997) CORINE biotopes. Ecole Nationale du 333 Génie Rural, des Eaux et des Forêts, Nancy.

334 Conservatoire botanique national du Bassin parisien (CBNBP), Muséum national

335 d'Histoire naturelle 2008. URL : http://cbnbp.mnhn.fr/cbnbp/.

336 Convention on Biological Diversity in Rio 1992. URL :

337 http://www.cbd.int/convention/convention.shtml

338 De Solla SR, Shirose LJ, Fernie KJ, Barrett GC, Brousseau CS, Bishop CA (2005)

339 Effect of sampling effort and species detectability on volunteer based anuran

340 monitoring programs. Biol. Conserv. 121: 585-594.

341 Estevez I, Christman MC (2006) Analysis of the movement and use of space of 342 animals in confinement: The effect of sampling effort. Appl. Anim. Behav. Sci. $34397: 221-240$.

344 Firbank LG, Barr CJ, Bunce RGH, et al (2003) Assessing stock and change in land 345 cover and biodiversity in GB: an introduction to Countryside Survey 2000. J. $346 \quad$ Environ. Manage. 67: 207-218.

347 Global Biodiversity Information Facility . 2008. URL : http://www.gbif.org

348 Haines-Young R, Barr CJ, Firbank LG, et al (2003) Changing landscapes, habitats 349 and vegetation diversity across Great Britain. J. Environ. Manage. 67: 267-281.

350 Haines-Young RH, Barr CJ, Black HIJ, et al (2000) Accounting for nature: assessing 351 habitats in the UK countryside. DETR, London. 
Biodiversity studies

352 Hintermann U, Weber D, Zangger A, Schmill J (2002) Biodiversity Monitoring in 353 Switzerland, BDM - Interim Report. Swiss Agency for the Environment, Forests $354 \quad$ and Landscape SAEFL.

355 IAURIF 2003. Institute for Planning and Development of the Paris Ile-de-France

$356 \quad$ Region. URL: http://www.iaurif.org

357 INSEE 2006. National Institute for Statistics and Economic Studies. http://www.insee.fr 358 Julliard R, Jiguet F, Couvet D (2003) Common birds facing global changes: what 359 makes a species at risk? Glob. Change Biol. 10: 148-154.

360 May RM (2002) The future of biological diversity in a crowded world. Current Science 82: $1325-1331$.

362 Metcalfe-Smith J, Di Maio J, Staton S, Mackie G (2000) Effect of sampling effort on the efficiency of the timed search method for sampling freshwater mussel communities. J. N. Am. Benthol. Soc. 19: 725-732.

Muratet A, Porcher E, Devictor V, et al (2008) Evaluation of floristic diversity in urban areas as a basis for habitat management. Applied Vegetation Science 11: 451-460.

Newson SE, Woodburn R, Noble DG, Baillie SR (2005) Evaluating the Breeding Bird Survey for producing national population size and density estimates. Bird Study 52 (1), 42-54.

Plattner M, Birrer S, Weber D (2004) Data quality in monitoring plant species richness in Switzerland. Community Ecology, 5, 135-143.

372 R Development, Core Team. 2007. R: A language and environment for statistical computing. R Foundation for Statistical Computing, Vienna. 
Biodiversity studies

376 Richardson DM, Pyšek P, Rejmánek M, Barbour MG, Panetta D, West CJ (2000)

377 Naturalization and invasion of alien plants: concepts and definitions. Diversity \& 378 Distributions 6: 93-107.

379 Salafsky N, Margoluis R, Redford KH, Robinson JG (2002) Improving the Practice

380 of Conservation: a Conceptual Framework and Research Agenda for 381 Conservation Science. Conserv. Biol. 16: 1469-1479.

382 Sauer JR, Hines JE, Gough G, Thomas I, Peterjohn BG (1997) The North American 383 Breeding Bird Survey Results and Analysis. Version 96.4. Patuxent Wildlife $384 \quad$ Research Center, Laurel, MD.

385 Walther B, Martin J-L (2001) Species richness estimation of bird communities: how 386 to control for sampling effort? Ibis 143: 413-419.

387 Weber D, Hintermann U, Zangger A (2004) Scale and trends in species richness:

388 considerations for monitoring biological diversity for political purposes. Glob. $389 \quad$ Ecol. Biogeogr. 13: 97-104.

390 Worm B, Duffy JE (2003) Biodiversity, productivity and stability in real food webs.

391 Trends in Ecology \& Evolution, 18(12): 628-632.

392

393 
Biodiversity studies

\section{Tables}

395

396 Table 1 Description of habitats types. The distribution of the number of inventories

397 across habitat types between 2001 and 2005 and the spatial distribution of habitats

398 are given.

399

\begin{tabular}{|c|c|c|}
\hline HABITAT type & $\begin{array}{l}\text { Number of visits } \\
\text { by surveyors }\end{array}$ & $\begin{array}{l}\text { Proportion of the total study area } \\
\text { (\%) (IAURIF 2003) }\end{array}$ \\
\hline Stagnant fresh water & 412 & \multirow{2}{*}{$1.2 \%$} \\
\hline Circle of water edges & 437 & \\
\hline Mesophile meadows & 259 & not available \\
\hline Deciduous forests & 2072 & $20.5 \%$ \\
\hline $\begin{array}{l}\text { Cultures (essentially } \\
\text { cereals) }\end{array}$ & 257 & $51.2 \%$ \\
\hline Urban parks and gardens & 1012 & $4 \%$ \\
\hline Cities and industrial sites & 1596 & $15.6 \%$ \\
\hline Wastelands & 1313 & $0.36 \%$ \\
\hline
\end{tabular}

400

401 
Biodiversity studies

402 Table 2 Result of the analysis of variance, where site richness was a function of (1)

403 habitat type (2) inventory month, (3) inventory year, and (4) all pairwise interactions.

404

Degree of 405

\begin{tabular}{llll} 
Parameters & freedom & F value & $\operatorname{Pr}(>\mathrm{F})$ \\
\hline Habitat & 7 & 135.82 & $<10^{-4}$ \\
Month & 7 & 22.94 & $<10^{-4}$ \\
Year & 4 & 31.20 & $<10^{-4}$ \\
Habitat*month & 49 & 4.20 & $<10^{-4}$ \\
Habitat*year & 27 & 6.14 & $<10^{-4}$ \\
Month*year & 27 & 5.26 & $<10^{-4}$ \\
Habitat*month*year & 165 & 1.78 & $<10^{-4}$ \\
\hline
\end{tabular}

406

407 


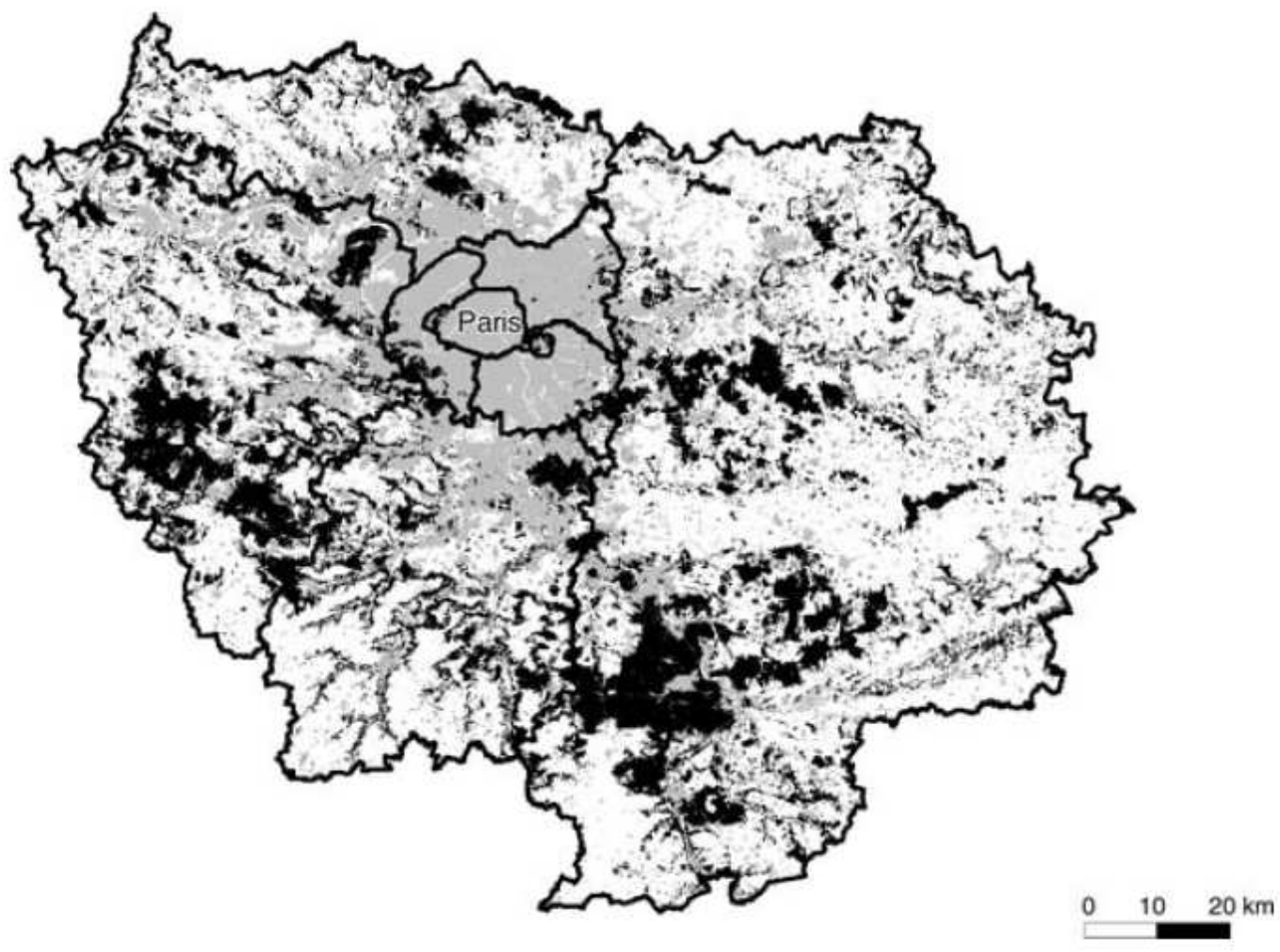

410 Fig. 1 Map of the study area, the Paris Ile-de-France region. Forests appear in black,

411 cultures and other rural habitats in white and open and built urban area in grey

412 (IAURIF 2003). Dark lines correspond to the district boundaries 
Biodiversity studies

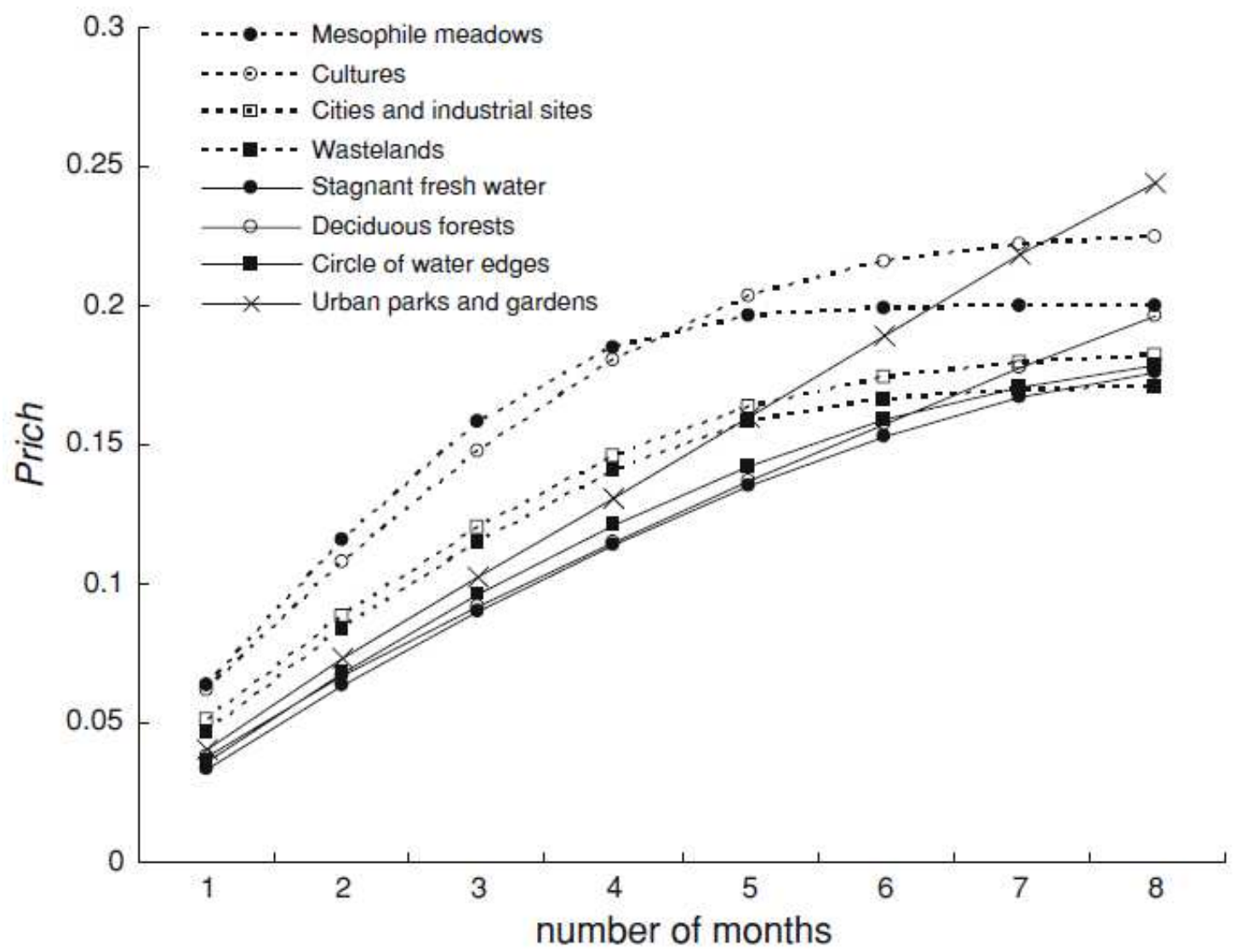

415

416 Fig. 2 Proportion of total species richness (Prich) as a function of the number of

417 seasons sampled (number of months)

418 
Biodiversity studies
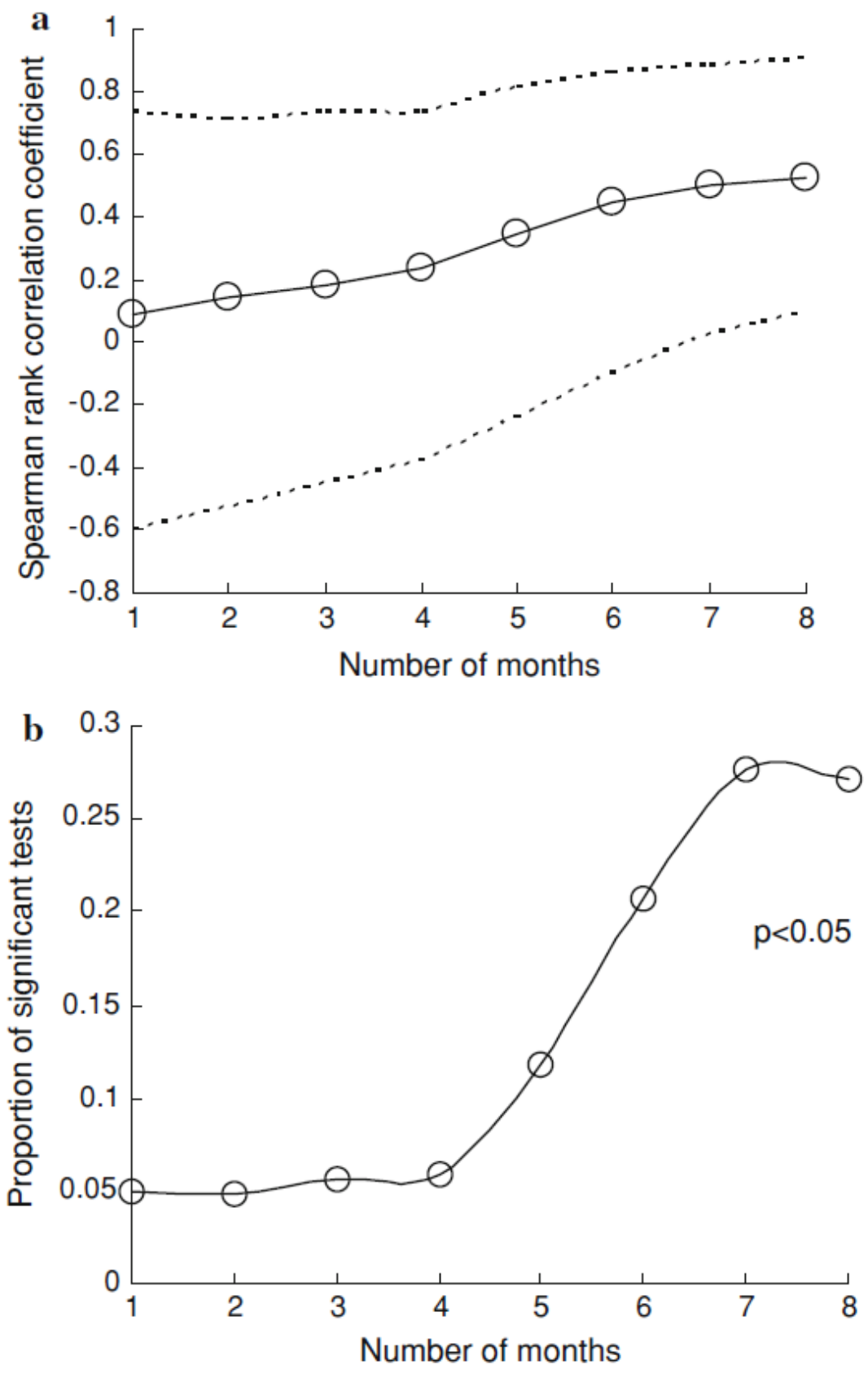

420 Fig. 3 Correlation between overall and recorded species richness in the different

421 habitats, as a function of the monitoring effort (increase of the number of inventory 422 months x). Protocol presented in method section. a Average (open circles) and 95\% 423 confidence intervals (dashed lines) Spearman coefficients of rank correlation $r_{s} . \mathrm{b}$ 424 Proportion of significant one-tailed correlations between overall and recorded species 425 richness among 50,000 independent computations of recorded species richness. 
Biodiversity studies

426

427

428

429

430

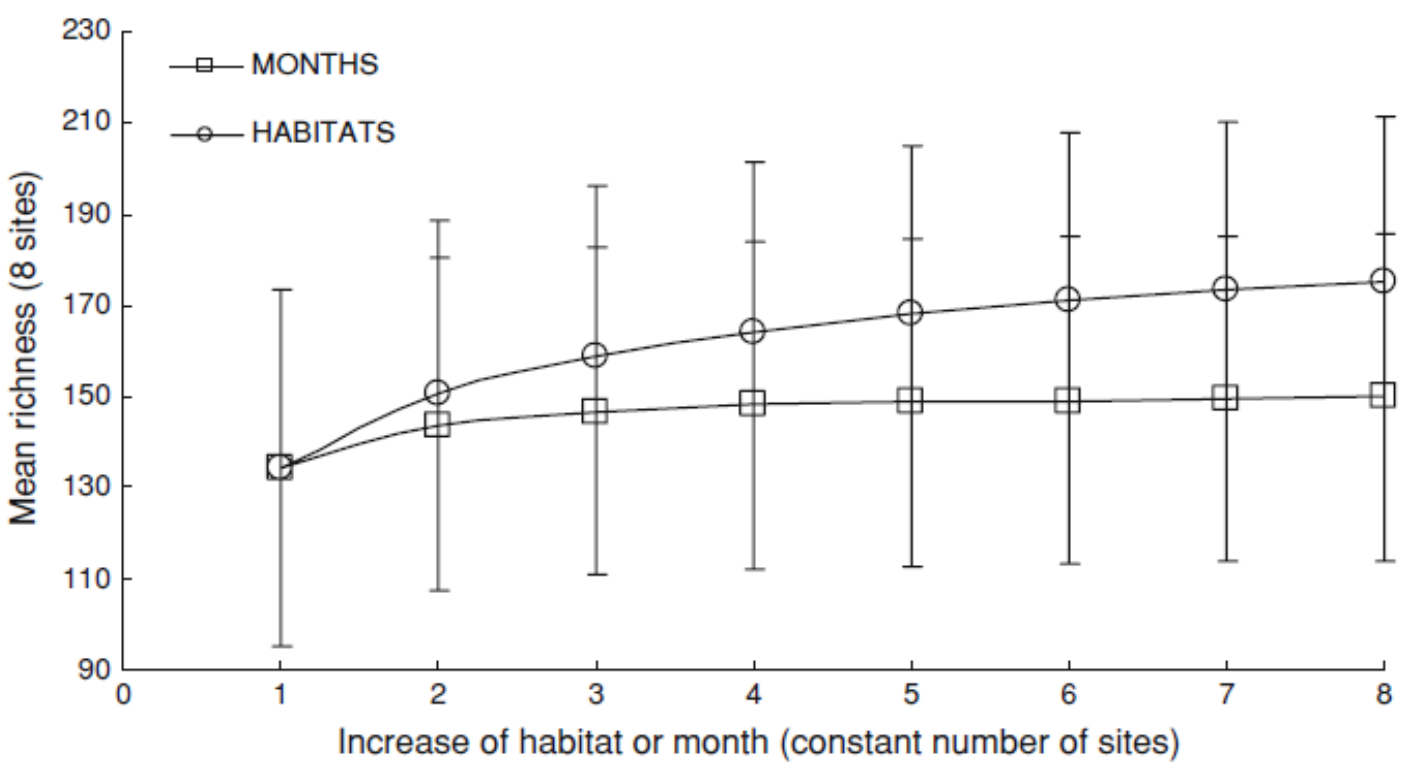

431

432 Fig. 4 Observed species richness as a function of the number of months or habitats

433 visited for a constant effort (eight sites sampled). Error bars represent standard errors. 